\title{
Evaluation of myocardial involvement in patients with connective tissue disorders: a multi-parametric cardiovascular magnetic resonance study
}

Agnes Mayr ${ }^{1}$, Daniel Kitterer ${ }^{2}$, Joerg Latus², Hannah Steubing ${ }^{3}$, Joerg Henes ${ }^{4}$, Francesco Vecchio ${ }^{3}$, Philipp Kaesemann ${ }^{3}$, Alexandru Patrascu ${ }^{3}$, Andreas Greiser ${ }^{5}$, Stefan Groeninger ${ }^{5}$, Niko Braun², M. Dominik Alscher ${ }^{2}$, Udo Sechtem ${ }^{3}$, Heiko Mahrholdt ${ }^{3 *}$ and Simon Greulich ${ }^{3}$

\begin{abstract}
Background: Severe arrhythmias or heart failure may be surrogates of myocardial involvement in patients with connective tissue disorders (CTD). However, most patients present with unspecific symptoms, normal ECG, and preserved left ventricular ejection fraction (LV-EF). Therefore, timely diagnosis by an accurate technique is crucial. Late gadolinium enhancement (LGE) cardiovascular magnetic resonance (CMR) has proven value for the detection of focal processes, but due to the often diffuse character of fibrosis/inflammation in CTD patients, CMR mapping techniques might be of incremental value for the assessment of myocardial involvement. Purpose of this study was to evaluate a multi-parametric CMR protocol as a screening tool for myocardial involvement in CTD patients. Methods: Forty CTD patients were prospectively enrolled and underwent CMR, twenty healthy volunteers served as control group.

Results: Mean LV-EF was 62 \%; LGE prevalence was low (18\%). CTD patients had higher native T1 (1008 vs. 962 ms, $p=0.001$ ), lower post contrast T1 (494 vs. $526 \mathrm{~ms}, p=0.008$ ), expanded extracellular volume (ECV) ( $28 \mathrm{vs} .25 \%, p=$ 0.001 ), and higher T2 values (53 vs. $49 \mathrm{~ms}, p<0.001$ ) compared to controls. Among patients with values higher than the $95 \%$ percentile of healthy controls, native T1 and T2 values seem to be the most promising discriminators.

Conclusion: CTD patients showed higher T1, ECV, and T2 values compared to controls, with most significant differences for native $\mathrm{T} 1$ and $\mathrm{T} 2$, which seem to be independent of the presence of LGE. Our data suggest that CMR mapping techniques are of incremental value in the detection of myocardial involvement in CTD patients.
\end{abstract}

Keywords: Connective tissue disorders, Myocardial involvement, CMR, LGE, Mapping

\section{Background}

Connective tissue disorders (CTD) are a heterogeneous form of rheumatic disorders comprising systemic lupus erythematosus (SLE), systemic sclerosis (SSc), Sjögren's syndrome, inflammatory muscle diseases and overlap syndrome [1]. There is a high variety in the prevalence of CTD, which may occur at all ages, but show a higher prevalence in young adults [1]. SLE is one of the most

\footnotetext{
* Correspondence: heiko.mahrholdt@rbk.de

${ }^{3}$ Division of Cardiology, Robert-Bosch-Medical Center Stuttgart,

Auerbachstrasse 110, 70376 Stuttgart, Germany

Full list of author information is available at the end of the article
}

common autoimmune disorders in the western world with a prevalence ranging from 15 to 50 per 100,000 persons [1]. Cardiovascular complications may manifest as inflammation of valves, myocardium, pericardium resulting in myocardial dysfunction, and heart failure [2]. The prevalence of SSc is estimated about 26 per 100,000 persons [1]. SSc is characterized by structural and functional abnormalities of small blood vessels, fibrosis of the skin and internal organs, activation of the immune system and autoimmunity [1]. Myocardial involvement often remains subclinical, however autopsy studies reveal diffuse myocardial fibrosis in up to $80 \%$ 
of cases [3-6], and sudden cardiac death occurs in up to $21 \%$ of SSc patients [5]. Therefore, timely detection of myocardial involvement in stages, which might be potentially reversible by an adequate treatment regimen, is of high clinical interest in patients with CTD.

Cardiovascular magnetic resonance (CMR) offers beside functional assessment excellent tissue characterization without the need of radiation. Recent data suggest that a CMR approach, including late gadolinium enhancement (LGE) for the detection of focal fibrosis, and T1 mapping sequences for the detection of diffuse fibrosis, might be useful in the detection of myocardial involvement in patients with SLE and SSc [2, 3]. However, for the assessment of inflammation, these groups used standard T2weighted images, which are known for severe limitations (e.g. proneness for artifacts) [7]. In the meantime, new T2 mapping sequences were developed, overcoming most of the standard T2-weighting limitations [8].

Consequently, aim of our study was to evaluate a comprehensive CMR protocol, including LGE and quantitative T1 and T2 mapping techniques for the assessment of both fibrosis and inflammation, as a screening tool for potential myocardial involvement in patients presenting with CTD.

\section{Methods}

\section{Patient population}

Forty patients presenting at our institution between October 2013 and March 2016 were consecutively enrolled if they fulfilled the following criteria: 1) connective tissue disorder; and 2) no history of CAD, myocardial infarction and/or prior revascularization; and 3) successfully underwent CMR imaging. Exclusion criteria were contraindications for CMR (e.g. pregnancy, pacemaker/ ICD, glomerular filtration rate $<30 \mathrm{ml} / \mathrm{min}$., previous adverse reactions to gadolinium, cochlea implant).

Healthy volunteers $(n=20)$ with no history of cardiac disease and free of symptoms served as control group. Prior to CMR, all participants provided a blood sample for measurement of hematocrit. The ethics committee of the University of Tuebingen approved the study and all patients gave written informed consent.

\section{CMR protocol}

ECG-gated CMR was performed in breath-hold using a 1.5 T Magnetom Aera (Siemens Healthcare, Erlangen, Germany) in line with current recommendations [9]. Both cine and LGE short axis images were prescribed every $10 \mathrm{~mm}$ (slice thickness $6 \mathrm{~mm}$ ) from base to apex. In-plane resolution was typically $1.2 \times 1.8 \mathrm{~mm}$. Cine was performed using a steady-state free-precession (SSFP) sequence. LGE images were acquired on average 510 min after contrast using a segmented inversion recovery gradient echo (IR-GRE)-sequence constantly adjusting inversion time to null normal myocardium [10, 11]. The contrast dose (Gadopentetate-Dimeglumine) was $0.15 \mathrm{mmol} / \mathrm{kg}$.

A modified look-locker inversion recovery prototype sequence (MOLLI) was used for T1 mapping and performed in a single midventricular short-axis (SAX) slice at mid-diastole, prior to and $20 \mathrm{~min}$ after administration of contrast, in line with current recommendations $[12,13]$.

Short axis T2 mapping was performed in a matching midventricular SAX before administration of contrast agent using an ECG-triggered T2-prepared single-shot bSSFP prototype sequence with multiple $\mathrm{T} 2$ preparation times [8].

More detailed information on $\mathrm{T} 1$ and $\mathrm{T} 2$ mapping sequences is provided in the Additional file 1.

\section{CMR analysis}

Cine and LGE images were evaluated by experienced observers (S.G., H.M.) as described elsewhere [14]. In brief, endocardial and epicardial borders were outlined on the short-axis cine images. Volumes, mass and ejectionfraction were derived by summation of epicardial and endocardial contours. Extent of LGE was assessed using QMass software (Medis, Leiden, The Netherlands), and the results were expressed as percentage of myocardial mass. The distribution of LGE was characterized as epicardial, intramural, transmural, or subendocardial [14].

Color-coded T1, ECV, and T2 maps were generated based on inline-generated, motion corrected raw images using QMap software 1.0 (Medis, Leiden, the Netherlands) in a single matching midventricular SAX. Motion-corrected T1 maps were examined for quality in three modalities: 1) raw T1 images 2) T1 maps 3) $\mathrm{R}^{2}$ maps. Endo- and epicardial contours were manually drawn by two experienced observers (S.G., A.M.), and then divided into 6 segments using the anterior right ventricular insertion point as reference. Care was taken to avoid partial volume effects at the endocardial and epicardial borders for T1, ECV and T2 maps. Global T1, $\mathrm{ECV}$, and T2 values were calculated: $\mathrm{T} 1$ values were determined by fitting an exponential model to the measured data [15]. Prior to CMR, the hematocrit was determined in all subjects, allowing with native and post contrast T1 measurements of the myocardium and blood pool the calculation of extracellular volume (ECV), using a previously described equation [16]. T2 results were obtained by fitting a 2-parameter intensityweighted exponential model (no offset term) [17].

\section{Variables and definitions}

All variables were collected directly from patients, and/ or medical records except CMR parameters, which were 
evaluated as described above. Most variables are selfexplanatory; all others are defined below.

Underlying connective tissue disorders had to fulfill the diagnostic criteria of the American College of Rheumatology or the European League Against Rheumatism, respectively. Due to the variety of CTD $(n=5)$, these were clustered into three subgroups:

1) $\mathrm{SSc}$

2) SLE

3) "Others": overlap syndrome, Sjögren's syndrome, and polymyositis.

Evaluation of disease activity in SSc and SLE patients: SSc: ESSG = European scleroderma study group [18].

SLE: SLEDAI = Systemic Lupus Erythematosus Disease Activity Index [19].

For SSc and SLE, subgroup analyses were performed. Due to the low number of patients and the heterogeneity of CTD in the "others" group (3 different CTD in 10 patients) no further subgroup analysis was performed.

\section{Statistical analysis}

Absolute numbers and percentages were computed to describe the patient population. All continuous variables were tested for normality using the Kolmogorov-Smirnov test. Normally distributed continuous variables were expressed as means (with standard deviation) and skewed variables were presented as medians (with quartiles). Comparisons between groups were made using the Mann-Whitney $U$ test or the Fisher's exact test, as appropriate. P-values (two-tailed) of $<0.05$ were considered significant. All statistical analyses were performed using SPSS, version 22.0 (IBM Corp., Armonk, NY, USA).

\section{Results}

\section{Patient characteristics}

In total $n=60$ subjects were included in the final analysis, see Table 1: $n=40$ patients with CTD, $n=20$ healthy individuals served as control group. At inclusion, CTD patients were $54 \pm 17$ years of age, predominantly female $(87 \%)$, and did not differ significantly from the control group, $p=0.10$ for age and $p=0.27$ for gender, respectively.

Most patients suffered from SSc $(n=17)$ or SLE $(n=$ $13)$. Others $(n=10)$ had overlap syndrome $(n=6)$, Sjögren's syndrome $(n=3)$, and polymyositis $(n=1)$. Nonspecific dyspnea and angina were the most frequently reported symptoms in the overall patient population (33 and $23 \%$, respectively). ECG abnormalities were detected in $n=8(20 \%)$ of the patients. In detail, $n=3$ showed left bundle branch block (in all of them CAD could be excluded by coronary angiography), $n=2$ had atrial fibrillation (one patient had coronary angiography and showed no CAD), $n=2$ had ventricular extrasystoles (in one of the patients CAD was ruled out by coronary angiography), $n=1$ patient showed a right bundle branch block. The majority (60\%) of our overall CTD population was on steroids during the time of CMR. Details are displayed in Table 1.

\section{General CMR results}

CMR findings can be viewed in Table 2. The mean LVEF was $62 \%$, and did not differ to our control group ( $p$ $=0.41$ ). Furthermore, functional CMR parameters (LV size, mass, etc.) were not significantly different between CTD patients and controls. LGE was present in 7 (18 \%) of the CTD patients, most commonly occurring in a non-ischemic pattern (epicardial and/or intramural) [14]. LGE was not present in any of the controls.

Looking at the SSc and SLE subgroups (Tables 3 and 4) revealed that mean LV-EF was also preserved, and the prevalence of LGE tended to be low (12\% SSc, $23 \%$ in SLE).

\section{$\mathrm{T} 1$ and ECV results}

We found higher native $\mathrm{T} 1$ values in the CTD patient population: 1008 (990-1042) ms vs. 962 (947-987) ms in controls, $p=0.001$; Table 2, Fig. 1a. Post contrast T1 values were decreased in comparison to controls: 494 (477-522) ms vs. 526 (508-553) ms, $p=0.008$, Table 2, Fig. 1b. For T1-derived ECV measures, CTD patients demonstrated significantly higher values: 28 (26-31) \% vs. 25 (24-27) \% in the control group, $p=0.001$, Table 2 , Fig. 1c. LGE-positive CTD patients had no significant differences in their native, post T1 values, and ECV results as compared to LGE-negative CTD patients ( $p=$ $0.36, p=0.63, p=0.76$, respectively).

Subgroup analysis of the SSc and SLE patients revealed higher T1 native and ECV values in SSc patients (1031 (1007-1075) ms, and 31 (28-34) \%, respectively), compared to $1002(976-1015) \mathrm{ms}$ and $26(25-29) \%$ in SLE patients, both $p<0.01$, also see Tables 3 and 4 . Furthermore, post contrast T1 values were lower in SSc patients: 494 (474-525) vs. SLE patients: 507 (479-539). However, this difference was not statistically significant $(p=0.43)$. Compared to healthy controls, patients with SSc demonstrated: 1$)$ significantly higher median native $\mathrm{T} 1$ values and ECV values (both $p<0.001), 2$ ) significantly decreased post contrast values $(p=0.02)$. Patients with SLE showed increased median T1 native values in comparison to healthy controls $(p=0.03)$. However, although increased, ECV values did not differ significantly to controls $(p=0.24)$. Furthermore, SLE patients demonstrated lower post contrast values than controls without reaching significance $(p=0.16)$. Figure 2 displays a LGE- 
Table 1 Baseline characteristics

\begin{tabular}{|c|c|c|c|c|}
\hline & All patients & $\mathrm{SLE}^{\mathrm{a}}$ & $\mathrm{SSC}^{\mathrm{a}}$ & Other CTD \\
\hline & $n=40$ & $n=13$ & $n=17$ & $n=10$ \\
\hline Age (yrs) & $54 \pm 17$ & $45 \pm 16$ & $55 \pm 16$ & $62 \pm 16$ \\
\hline Gender (male) & $5(13 \%)$ & $3(23 \%)$ & $1(6 \%)$ & $1(10 \%)$ \\
\hline \multicolumn{5}{|l|}{ Diagnosis } \\
\hline Systemic lupus erythematosus & $13(33 \%)$ & $13(100 \%)$ & - & - \\
\hline Systemic sclerosis & $17(38 \%)$ & - & $17(100 \%)$ & - \\
\hline Overlap syndrome & $6(15 \%)$ & - & - & $6(60 \%)$ \\
\hline Sjögren's syndrome & $3(7 \%)$ & - & - & $3(30 \%)$ \\
\hline Polymyositis & $1(2 \%)$ & - & - & $1(10 \%)$ \\
\hline \multicolumn{5}{|l|}{ Cardiovascular risk factors } \\
\hline Diabetes & $1(2 \%)$ & - & - & $1(10 \%)$ \\
\hline Hypertension & $13(33 \%)$ & $4(33 \%)$ & $4(23 \%)$ & $5(50 \%)$ \\
\hline Smoking $^{b}$ & $14(35 \%)$ & $6(46 \%)$ & $7(41 \%)$ & $1(10 \%)$ \\
\hline Hyperlipidemia & $7(18 \%)$ & $1(8 \%)$ & $2(12 \%)$ & $4(40 \%)$ \\
\hline Family history of CVD & $14(35 \%)$ & $6(46 \%)$ & $4(23 \%)$ & $4(40 \%)$ \\
\hline Obesity (BMI $\geq 30$ kg/m²) & $4(10 \%)$ & $2(15 \%)$ & $2(12 \%)$ & - \\
\hline \multicolumn{5}{|l|}{ Symptoms (multiple possible) } \\
\hline Angina & $9(23 \%)$ & $3(23 \%)$ & $2(12 \%)$ & $4(40 \%)$ \\
\hline Dyspnea & $13(33 \%)$ & $3(23 \%)$ & $6(35 \%)$ & $4(40 \%)$ \\
\hline Palpitations & $3(7 \%)$ & $1(8 \%)$ & - & $2(20 \%)$ \\
\hline Syncope & $1(2 \%)$ & - & - & $1(10 \%)$ \\
\hline ECG abnormality & $8(20 \%)$ & $1(8 \%)$ & $3(18 \%)$ & $4(40 \%)$ \\
\hline \multicolumn{5}{|l|}{ Years since diagnosis } \\
\hline$<1$ & $8(20 \%)$ & $5(38.5 \%)$ & $1(6 \%)$ & $2(20 \%)$ \\
\hline $1-4$ & $12(30 \%)$ & $2(15 \%)$ & $9(53 \%)$ & $1(10 \%)$ \\
\hline $5-9$ & $6(15 \%)$ & $1(8 \%)$ & $3(18 \%)$ & $2(20 \%)$ \\
\hline$\geq 10$ & $14(35 \%)$ & $5(38.5 \%)$ & $4(23 \%)$ & $5(50 \%)$ \\
\hline \multicolumn{5}{|l|}{ Disease activity } \\
\hline SLEDAI & - & $16(6-23)$ & - & - \\
\hline ESSG & - & - & $3.5(1.4-5.5)$ & - \\
\hline Hematocrit & $0.38(0.34-0.40)$ & $0.38(0.34-0.40)$ & $0.38(0.34-0.42)$ & $0.38(0.37-0.39)$ \\
\hline \multicolumn{5}{|l|}{ Medication } \\
\hline Beta-blockers & $6(15 \%)$ & $4(31 \%)$ & - & $2(20 \%)$ \\
\hline ARB & 19 (48 \%) & $5(39 \%)$ & 9 (53 \%) & $5(50 \%)$ \\
\hline ASA & $6(15 \%)$ & $1(8 \%)$ & $2(12 \%)$ & $3(30 \%)$ \\
\hline$C C B$ & $6(15 \%)$ & $1(8 \%)$ & $4(23 \%)$ & $1(10 \%)$ \\
\hline Statins & $7(18 \%)$ & $3(23 \%)$ & $2(12 \%)$ & $2(20 \%)$ \\
\hline Diuretics & $6(15 \%)$ & $3(23 \%)$ & 2 (12 \%) & 1 (10\%) \\
\hline Steroids & 24 (60 \%) & 10 (77 \%) & $8(47 \%)$ & $6(60 \%)$ \\
\hline NSAID & $1(2 \%)$ & $1(8 \%)$ & - & - \\
\hline Chloroquines & $4(10 \%)$ & $2(15 \%)$ & $1(6 \%)$ & 1 (10 \%) \\
\hline Antibodies & $1(2 \%)$ & $1(8 \%)$ & - & - \\
\hline
\end{tabular}


Table 1 Baseline characteristics (Continued)

\begin{tabular}{|c|c|c|c|c|}
\hline Cyclophosphamide & $9(23 \%)$ & 5 (39\%) & $3(18 \%)$ & $1(10 \%)$ \\
\hline Azathioprine & $3(8 \%)$ & $2(15 \%)$ & - & $1(10 \%)$ \\
\hline Methotrexate & $1(2 \%)$ & - & - & $1(10 \%)$ \\
\hline
\end{tabular}

All values are $\mathrm{n}(\%)$ or mean $\pm \mathrm{SD}$ or interquartile ranges

SLE systemic lupus erythematosus, SSC systemic sclerosis, CTD connective tissue disease, CVD cardiovascular disease, $B M I$ body mass index, $E C G$ electrocardiogram, $S L E D A /$ systemic lupus erythematosus disease activity index, $A C R$ American College of Rheumatology, ESSG European scleroderma study group, $A R B$ angiotensin receptor blockers, $A S A$ acetylsalicylic acid, CCB calcium channel blockers, NSAID nonsteroidal anti-inflammatory drug

a percentages based on number of SLE patients/SSC patients, respectively

${ }^{\mathrm{b} C u r r e n t}$ or ever-smokers

negative female SLE patient showing increased native $\mathrm{T} 1, \mathrm{ECV}$ and $\mathrm{T} 2$, and decreased post contrast $\mathrm{T} 1$ values.

In our patients with ECG abnormalities, native and post $\mathrm{T} 1$ values, and ECV were not significantly different to the values in patients with normal ECG ( $p=0.73, p=$ $0.65, p=0.93$, respectively).

\section{T2 results}

Median myocardial T2 values were significantly higher in patients with CTD than in controls: 53 (52-58) ms vs. 49 (48-51) ms, $p<0.001$, Table 2, Fig. $1 \mathrm{~d}$. This difference remained significant independent of the patients' LGE status; LGE-negative patients: $54(51-58) \mathrm{ms}, p \leq$

Table 2 CMR findings

\begin{tabular}{|c|c|c|c|}
\hline & Controls $(n=20)$ & Patients $(n=40)$ & $P$ \\
\hline LV-EF (\%) & $66 \pm 4$ & $62 \pm 12$ & 0.41 \\
\hline LV-EDV (ml) & $109 \pm 28$ & $108 \pm 36$ & 0.76 \\
\hline LV-ESV (ml) & $37 \pm 13$ & $41 \pm 25$ & 0.86 \\
\hline LV-SV & $71 \pm 16$ & $66 \pm 13$ & 0.41 \\
\hline LV-EDD & $45 \pm 5$ & $46 \pm 5$ & 0.67 \\
\hline $\mathrm{LA}\left(\mathrm{cm}^{2}\right)$ & $20 \pm 4$ & $20 \pm 5$ & 0.68 \\
\hline IVS (mm) & $10 \pm 2$ & $9 \pm 3$ & 0.63 \\
\hline $\mathrm{PA}(\mathrm{mm})$ & $24 \pm 4$ & $24 \pm 6$ & 0.56 \\
\hline LV mass (g) & $76 \pm 18$ & $82 \pm 24$ & 0.50 \\
\hline LGE per patient & - & $7(18 \%)$ & \\
\hline Epicardial & - & $1(2 \%)$ & \\
\hline Intramural & - & $6(15 \%)$ & \\
\hline Transmural & - & $1(2 \%)$ & \\
\hline Subendocardial & - & - & \\
\hline$\%$ LV mass & - & 5.3 & \\
\hline Native T1 (ms) & 962 (947-987) & 1008 (990-1042) & 0.001 \\
\hline Post contrast T1 (ms) & $526(508-553)$ & $494(477-522)$ & 0.008 \\
\hline ECV (\%) & $25(24-27)$ & $28(26-31)$ & 0.001 \\
\hline $\mathrm{T} 2$ (ms) & $49(48-51)$ & $53(52-58)$ & $<0.001$ \\
\hline
\end{tabular}

All values are $\mathrm{n}$ or mean $\pm \mathrm{SD}$ or interquartile ranges

$C M R$ cardiac magnetic resonance, $L V$ left ventricular, $E F$ ejection fraction, $E D V$ end-diastolic volume, ESV end-systolic volume, SV stroke volume, $E D D$ enddiastolic diameter, LA left atrium, IVS interventricular septum, PA pulmonary artery, $L G E$ late gadolinium enhancement, $E C V$ extracellular volume
0.001, and LGE-positive patients: $52(52-58) \mathrm{ms}, p=$ 0.001 .

In the subgroup analysis, SSc patients had higher T2 values than patients with SLE: 58 (54-59) ms vs. 51 (4953) $\mathrm{ms}, p=0.001$. However, both (SSc and SLE) differed significantly to the control group, $p<0.001, p=0.02$, respectively, Tables 3 and 4 .

These findings are illustrated by Fig. 3, displaying a LGE negative SSc patient with severely increased values for native T1, ECV and T2, and decreased values for post contrast $\mathrm{T} 1$ in comparison to controls.

T2 values in patients with ECG abnormalities were not significantly different from T2 values in patients with normal ECG, $p=0.60$.

Table 3 CMR findings systemic sclerosis (SSC)

\begin{tabular}{|c|c|c|c|}
\hline & Controls $(n=20)$ & Patients $(n=17)$ & $P$ \\
\hline LV-EF (\%) & $66 \pm 4$ & $62 \pm 16$ & 0.48 \\
\hline LV-EDV (ml) & $109 \pm 28$ & $105 \pm 29$ & 0.82 \\
\hline LV-ESV (ml) & $37 \pm 13$ & $38 \pm 14$ & 0.87 \\
\hline LV-SV & $71 \pm 16$ & $67 \pm 16$ & 0.63 \\
\hline LV-EDD & $45 \pm 5$ & $46 \pm 4$ & 0.42 \\
\hline $\mathrm{LA}\left(\mathrm{cm}^{2}\right)$ & $20 \pm 4$ & $20 \pm 5$ & 0.93 \\
\hline IVS (mm) & $10 \pm 2$ & $10 \pm 4$ & 0.76 \\
\hline $\mathrm{PA}(\mathrm{mm})$ & $24 \pm 4$ & $26 \pm 8$ & 0.81 \\
\hline LV mass (g) & $76 \pm 18$ & $81 \pm 31$ & 0.82 \\
\hline LGE per patient & - & $2(12 \%)$ & \\
\hline Epicardial & - & - & \\
\hline Intramural & - & $2(12 \%)$ & \\
\hline Transmural & - & - & \\
\hline Subendocardial & - & - & \\
\hline$\%$ LV mass & - & 1.3 & \\
\hline Native T1 (ms) & 962 (947-987) & 1031 (1007-1075) & $<0.001$ \\
\hline Post contrast T1 (ms) & $526(508-553)$ & $494(474-525)$ & 0.02 \\
\hline ECV (\%) & $25(24-27)$ & $31(28-34)$ & $<0.001$ \\
\hline T2 (ms) & $49(48-51)$ & $58(54-59)$ & $<0.001$ \\
\hline
\end{tabular}

All values are $\mathrm{n}$ or mean $\pm \mathrm{SD}$ or interquartile ranges

$C M R$ cardiac magnetic resonance, $L V$ left ventricular, $E F$ ejection fraction, $E D V$ end-diastolic volume, ESV end-systolic volume, SV stroke volume, EDD enddiastolic diameter, $L A$ left atrium, IVS interventricular septum, $P A$ pulmonary artery, $L G E$ late gadolinium enhancement, $E C V$ extracellular volume 
Table 4 CMR findings systemic lupus erythematosus (SLE)

\begin{tabular}{|c|c|c|c|}
\hline & Controls $(n=20)$ & Patients $(n=13)$ & $P$ \\
\hline LV-EF (\%) & $66 \pm 4$ & $63 \pm 7$ & 0.60 \\
\hline LV-EDV (ml) & $109 \pm 28$ & $111 \pm 36$ & 1 \\
\hline LV-ESV (ml) & $37 \pm 13$ & $43 \pm 24$ & 0.82 \\
\hline LV-SV & $71 \pm 16$ & $67 \pm 12$ & 0.55 \\
\hline LV-EDD & $45 \pm 5$ & $46 \pm 4$ & 1 \\
\hline $\mathrm{LA}\left(\mathrm{cm}^{2}\right)$ & $20 \pm 4$ & $17 \pm 3$ & 0.02 \\
\hline IVS (mm) & $10 \pm 2$ & $9 \pm 1$ & 0.35 \\
\hline PA (mm) & $24 \pm 4$ & $22 \pm 3$ & 0.08 \\
\hline LV mass (g) & $76 \pm 18$ & $85 \pm 21$ & 0.41 \\
\hline LGE per patient & - & $3(23 \%)$ & \\
\hline Epicardial & - & $1(8 \%)$ & \\
\hline Intramural & - & $2(15 \%)$ & \\
\hline Transmural & - & $1(8 \%)$ & \\
\hline Subendocardial & - & - & \\
\hline$\%$ LV mass & - & 6.2 & \\
\hline Native T1 (ms) & 962 (947-987) & 1002 (976-1015) & 0.03 \\
\hline Post contrast $\mathrm{T} 1$ (ms) & $526(508-553)$ & 507 (479-539) & 0.16 \\
\hline ECV (\%) & $25(24-27)$ & $26(25-29)$ & 0.24 \\
\hline T2 (ms) & $49(48-51)$ & $51(49-53)$ & 0.02 \\
\hline
\end{tabular}

All values are $\mathrm{n}$ or mean $\pm \mathrm{SD}$ or interquartile ranges

$C M R$ cardiac magnetic resonance, $L V$ left ventricular, $E F$ ejection fraction, $E D V$ end-diastolic volume, ESV end-systolic volume, SV stroke volume, EDD enddiastolic diameter, $L A$ left atrium, IVS interventricular septum, $P A$ pulmonary artery, $L G E$ late gadolinium enhancement, ECV extracellular volume

\section{Values above the $95 \%$ percentile of normal}

Defining the $95 \%$ percentile of our control group as a threshold for definite abnormal values, we found values above $1033 \mathrm{~ms}$ for native T1, below $451 \mathrm{~ms}$ for post contrast $\mathrm{T} 1$, above $32 \%$ for $\mathrm{ECV}$, and above $54 \mathrm{~ms}$ for T2 to be abnormal, see Fig. 4.

$25 \%(n=10)$ of the CTD patients demonstrated a native T1 value above the $95 \%$ percentile of the matched control group: $n=9$ patients were LGE-negative, $n=1$ patient was LGE- positive, also see Fig. 4a. In $n=3$ patients, post contrast values were below $455 \mathrm{~ms}$, all of them were reported LGE-negative, see Fig. 4b. Measurement of ECV revealed that $n=8$ patients had definite abnormal values ( $n=7$ LGE-negative, $n=1$ LGEpositive), see Fig. 4c. Almost $40 \%$ (15 out of 40 CTD patients) showed definite abnormal T2 values: $n=13$ LGE-negative, $n=2$ LGE-positive, see Fig. $4 \mathrm{~d}$.

Almost $50 \%$ of SSc patients (8 out of $17 \mathrm{SSc}$ ) had an increased native $\mathrm{T} 1$ value above the $95 \%$ percentile of controls, and only one of these was LGE-positive. Three patients (all LGE-negative) had post contrast T1 values lower, and six patients (5 LGE-negative, 1 LGE-positive) had ECV values higher than the $95 \%$ percentile of controls. $65 \%$ of the SSc patients demonstrated definitely abnormal T2 values: $n=10$ were LGE-negative, $n=1$ LGE-positive, also see Fig. 5.

In the SLE subgroup, values higher than the $95 \%$ percentile of controls were found in $n=3$ patients (23\%), with one patient showing both increased T2 and native T1 values beyond the $95 \%$ percentile of normal, and the two other patients isolated increased ECV or T2 beyond the $95 \%$ percentile of normal, respectively. Only one of these patients was reported LGE-positive, also see Fig. 5.

\section{Discussion}

To the best of our knowledge, this is the first study evaluating cardiac involvement in patients with CTD and preserved left ventricular ejection fraction by a comprehensive CMR approach, including LGE CMR, as well as T1 and T2 mapping techniques. The findings are as follows: 1) Patients with CTD show increased native T1, ECV, T2 and decreased post contrast T1 values compared to controls. 2) Subgroup analysis of SSc and SLE patients revealed that native $\mathrm{T} 1$ and $\mathrm{T} 2$ values seem to be higher in patients with SSc compared to patients with SLE. However, both parameters can separate between SSc/SLE patients and controls. 3) Abnormal values beyond the $95 \%$ percentile of healthy controls might help to detect myocardial involvement in patients with CTD even in the absence of LGE.

\section{Patient characteristics and general CMR results}

Most patients were middle-aged and female, in line with previous reports [3]. The majority of patients was nonor oligosymptomatic, and had normal ECG, underlining that the diagnosis of cardiac involvement is a challenge in CTD, Table 1. The mean LV-EF in our cohort was preserved (62\%), cardiac dimensions did not differ from controls, Table 2. LGE was present in $18 \%$ of the CTD patients, occurring in a non-ischemic pattern in accordance with other studies [2, 3, 20-22].

\section{$\mathrm{T} 1$ and ECV results}

We found higher native T1 values and increased ECV in our CTD population in comparison to controls, Table 2, Fig. $1 \mathrm{a}+\mathrm{c}$. Furthermore, post contrast T1 values were decreased in comparison to controls, Table 2, and Fig. $1 \mathrm{~b}$. Since these differences are independent of the presence of LGE, they may allow early detection of subclinical myocardial alterations in patients with CTD, as reported in other inflammatory cardiomyopathies [23, 24].

In the SSc subgroup, differences for native $\mathrm{T} 1$ and ECV were even larger than in the overall CTD population, suggesting a high rate of diffuse myocardial involvement detected by T1 mapping, supporting data from Ntusi et al. [3], who found also elevated native T1 and ECV in SSc patients. At first sight, the mapping data in this study seem to conflict with the low prevalence of 

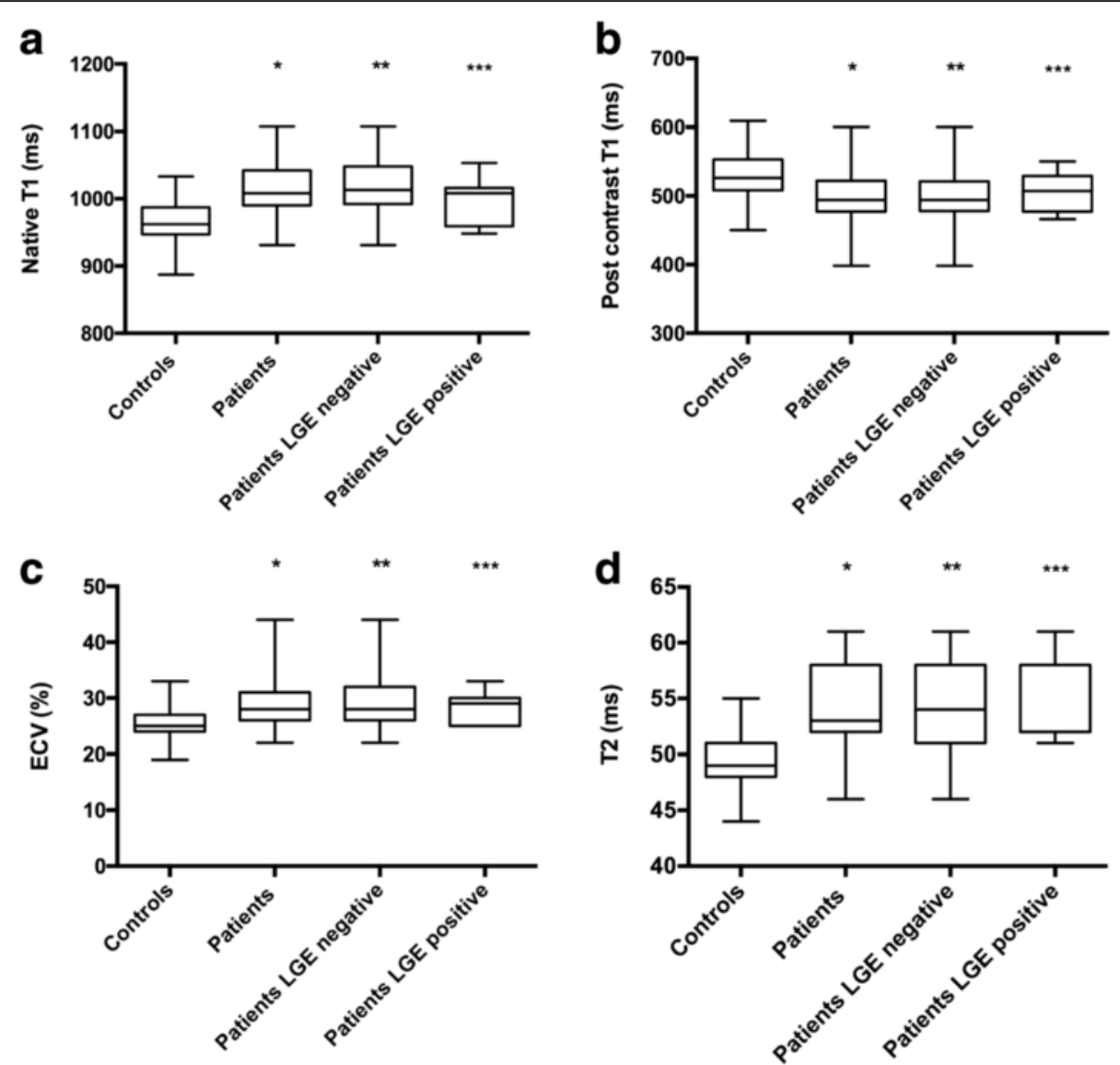

Fig. 1 Box plots for median native T1 (a), post contrast T1 (b), extracellular volume fraction (ECV; $\mathbf{c}$ ), and T2 mapping (d) in controls, all CTD patients, late gadolinium enhancement (LGE) negative CTD patients, and LGE positive CTD patients; the center line in each box represents the median, whereas the lower and upper limits of each box represent the 25th and 75th percentiles, respectively. CTD patients (all, LGE negative, LGE positive except for native T1 and post contrast T1, respectively) showed values which were significantly different to the values of the control group; $* * * * * * *$ each $p \leq 0.05)$

LGE (12 \%). However, LGE has its strengths in detecting focal processes (e.g. infarcted myocardium vs. remote myocardium), whereas in diffuse processes this technique is of limited value. Conversely, mapping techniques, which provide absolute quantitative values, rather than just visual or semi-quantitative interpretation of the images, perform well in the assessment of diffuse myocardial processes [7]. Therefore, the T1 and ECV findings in this study might be the surrogate for the high rate of diffuse fibrosis (44-100\%) observed by endomyocardial biopsy or autopsy in SSc patients $[25,26]$, and might be a useful tool not only for detection of myocardial involvement, but also for evaluation of an adequate response to immunosuppressive agents during the clinical course of the disease.

In the SLE subgroup, we observed lower T1 and ECV differences to controls than in the SSc subgroup. Consequently, although showing increased ECV and decreased post contrast $\mathrm{T} 1$ values compared to controls, the difference was significant only for native T1 values, $p=0.03$.
This might have at least two reasons: 1) In contrast to SLE patients, autopsy studies from SSc patients revealed a high rate of diffuse fibrosis, which might be the surrogate for higher native T1 and ECV values in SSc patients [25, 26]. 2) Our finding that native T1 seems to separate best between SLE patients and healthy controls, is supported by a recent study [2], which identified native T1 a) as the best parameter to separate between SLE patients and controls, and b) as an independent predictor of the underlying SLE diagnosis. However, in the study by Puntmann [2] also post contrast $\mathrm{T} 1$ values and ECV differed significantly to the control group. They included 33 asymptomatic SLE patients, with an activity index (SLEDAI) of 0 , and observed a high LGE prevalence of $61 \%(n=20)$, which is in contrast to our study (SLEDAI 16, prevalence of LGE $23 \%$ ). Another explanation for these differences might be the time duration from SLE diagnosis to CMR imaging: In the study from Puntmann et al., the average time from SLE diagnosis to imaging was 


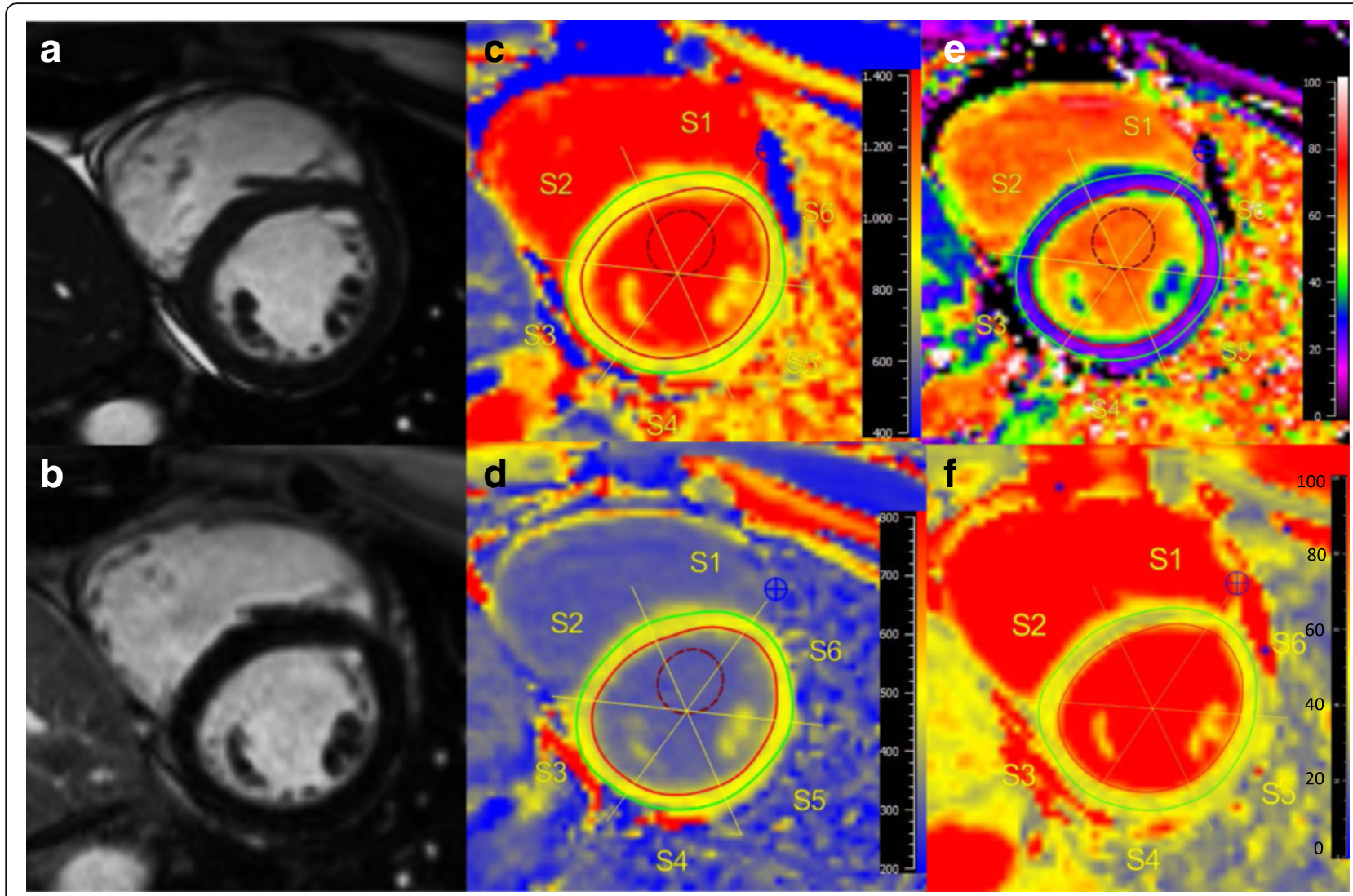

Fig. 2 Patient with SLE. Cardiovascular magnetic resonance (CMR) of a 30-year old female presenting with no symptoms and normal ECG for her sixth cycle of cyclophosphamide. 3 months ago, the diagnosis SLE was settled. Cine images (a) revealed a preserved LV-EF (63 \%), LGE images (b) demonstrated no enhancement. Native T1 map (c) showed an increased T1 with 999 ms (normal median range 962 (947-987) ms), decreased post-contrast T1 (d) with 489 ms (normal median range 526 (508-553) ms), and expanded ECV (e) of 27 \% (normal median range 25 (24-27) \%). T2 (f) was prolonged with 51 ms (normal median range 49 (48-51) ms)

7.4 years whereas in our study almost $40 \%$ had their CMR within the first year of SLE diagnosis. Therefore, it might be argued that the grade of diffuse fibrosis, as well as the presence of focal fibrosis detected by LGE, might increase in later stages of the disease. Since both studies found that native $\mathrm{T} 1$ is the most sensitive parameter to separate between SLE patients and controls, native T1 may play an important role in: a) initial diagnosis of myocardial involvement and b) the monitoring in SLE patients.

Our findings add knowledge to the potential role of T1 mapping in patients with different CTD, since this technique seems to provide more detailed tissue characterization than LGE alone. This might have clinical implications for the assessment of disease activity, and monitoring of the response to immunosuppressive medication in CTD patients. Moreover, since T1 and ECV values in patients with ECG abnormalities did not differ to the values of patients with normal ECG, the presence of ECG abnormalities alone may be of limited diagnostic value for detecting myocardial involvement in CTD patients.

\section{T2 results}

In contrast to $\mathrm{T} 1$ mapping, myocardial $\mathrm{T} 2$ values correlate closely with free tissue water content $[27,28]$, predisposing them for the assessment of active myocardial inflammation in systemic disorders such as CTD. Newer T2 mapping sequences provide objective and robust data $[8,29]$, and will most likely replace previously described T2-weighted sequences [7].

As expected in systemic inflammatory disorders such as CTD, median myocardial T2 values were significantly higher than in controls, suggesting myocardial involvement due to systemic inflammation, Table 2, Fig. 1d. Of note, $\mathrm{T} 2$ performs even better than native $\mathrm{T} 1$ to separate controls from CTD patients $(p<0.001, p=0.001$, respectively). This difference remains significant by dividing the CTD population in a LGE-positive and a LGE-negative group, underlining the additional value of T2 mapping in comparison to the performance of LGE CMR alone.

For the SSc subgroup, we found only studies in the literature that used T2-weighted images for the assessment of inflammation instead of newer T2 mapping techniques 


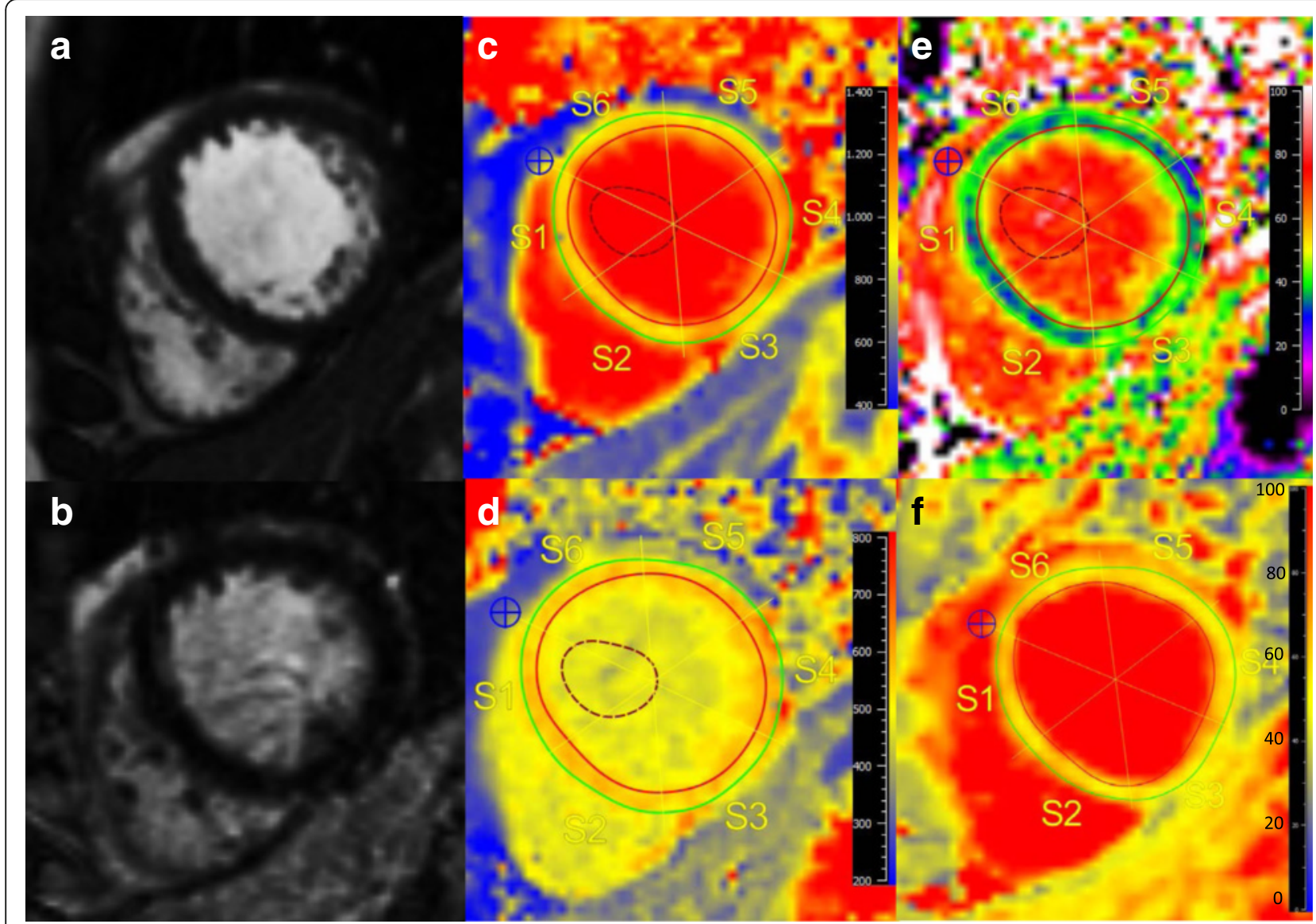

Fig. 3 CMR of a 45-year old female with a history of SSc since 15 years. She was suffering from nonspecific dyspnea, and palpitations. ECG was unremarkable. Cine CMR (a) revealed a preserved LV-EF (61 \%) with normal cardiac dimensions. LGE images (b) revealed no LGE. However, native T1 (c) with 1108 ms, ECV (e) with $40 \%$, and T2 (f) with 61 ms were severely increased, whereas post contrast T1 (540 ms) (d) was within normal range compared to our control group (median native T1 962 ms, ECV $25 \%$, post contrast T1 526 ms, T2 49 ms)

$[3,30]$. We filled this gap and found higher T2 values both than controls $(p<0.001)$, and patients with $\operatorname{SLE}(p=$ $0.001)$, suggesting a high grade of myocardial inflammation, possibly representing active disease, in SSc patients. The occurrence of both myocardial inflammation and diffuse fibrosis is a well-known finding in these patients [3]. Thus, a comprehensive CMR approach including LGE, T1 and T2 mapping seems a reasonable approach to evaluate both chronic and active stages of the disease in SSc patients.

Our data are also supported by a recent study [31], which reported elevated T2 values in SLE patients compared to controls. However, their T2 values were higher in SLE patients and controls as compared to the values in this study, which might have the following reasons: 1) Different patient populations: our patients were younger; 2) different grades of inflammation due to different immunosuppressive treatment regimen: $77 \%$ of our patients were on steroids vs. only $17 \%$ in the latter study. 3) Differences in the T2 mapping sequence and map analysis software. Therefore, as long as there are no consistent mapping sequences, each institution should create its individualized normal values [12]. Of note, T2 values of our control group were in line with the results of other groups [32].

Since increased T2 values are supposed to represent potentially reversible processes [31], T2 mapping might play an important role as a quantitative biomarker, which might serve as surrogate for response or failure of immunosuppressive agents.

As shown above for T1 values, T2 values in patients with normal vs. abnormal ECG did not differ significantly, underlining the need for further detailed tissue characterization for the detection of myocardial involvement in CTD.

\section{Values above the $95 \%$ percentile of normal}

Despite highly significant differences in $\mathrm{T} 1$ and $\mathrm{T} 2$ values between the CTD population and controls, there is still some overlap in values, hampering the diagnosis of myocardial involvement in the individual CTD patient, also see Fig. 1. Therefore, we used the $95 \%$ 


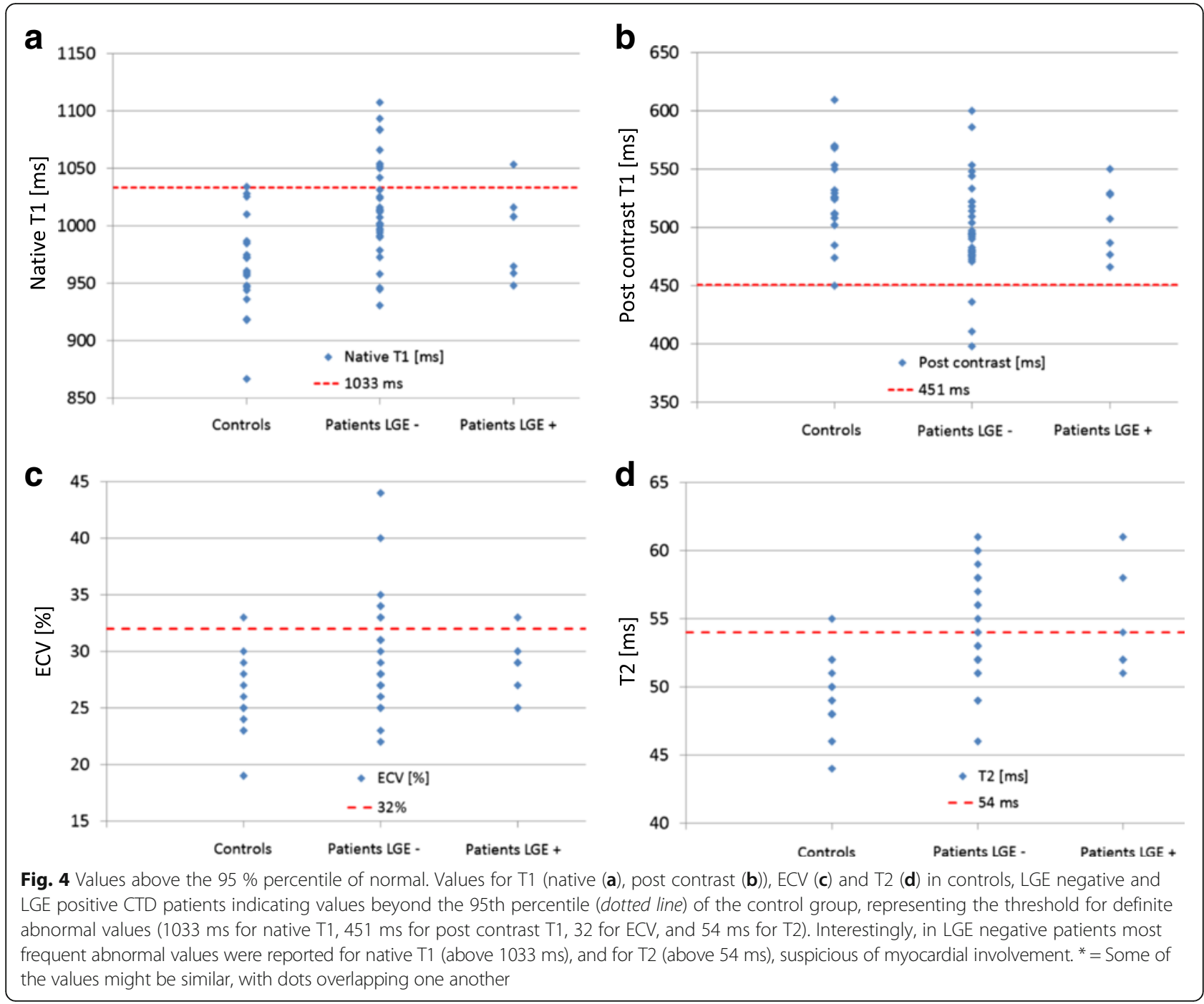

percentile of our control group as a threshold for definite abnormal values in patients with CTD.

The majority of abnormal values were reported for T2 $(n=15)$, and native T1 $(n=10)$, suggesting to be the most promising parameters for potential detection of myocardial involvement. Of note, $87 \%$ of these patients with elevated T2 values, and $90 \%$ of the patients with elevated T1 native values, were LGE-negative, see Fig. 4.

In the SSc and SLE subgroups we found comparable results, with native $\mathrm{T} 1$ and $\mathrm{T} 2$ as most frequent parameters above the $95 \%$ percentile of normal, and a high rate of LGE-negative patients, see Fig. 5. These findings underline the additional benefit of the newer mapping techniques compared to LGE imaging alone.

\section{Clinical implications}

In this study, we could demonstrate that mapping sequences in addition to LGE-CMR might be useful for the detection of myocardial involvement in patients with
CTD. Patients with CTD show higher T1, ECV, and T2 values compared to healthy controls. These findings are independent of the presence of LGE. Furthermore, subgroup analysis in SSc and SLE patients revealed that native T1 mapping and T2 mapping are the best parameters to separate between normal subjects and patients. This could be confirmed among patients with values higher than the $95 \%$ percentile of controls, suggesting a combination of both fibrosis and inflammation in CTD patients.

Despite potential life-threatening complications by myocardial involvement of CTD, many patients will present with nonspecific symptoms, normal ECG, and preserved LV-EF. Thus, a comprehensive CMR approach may be of future clinical importance not only for detection of myocardial involvement but also for response to treatment. Nevertheless, larger randomized trials are warranted to investigate the diagnostic and prognostic value of abnormal mapping findings, before these sequences can be implemented in the clinical routine. 


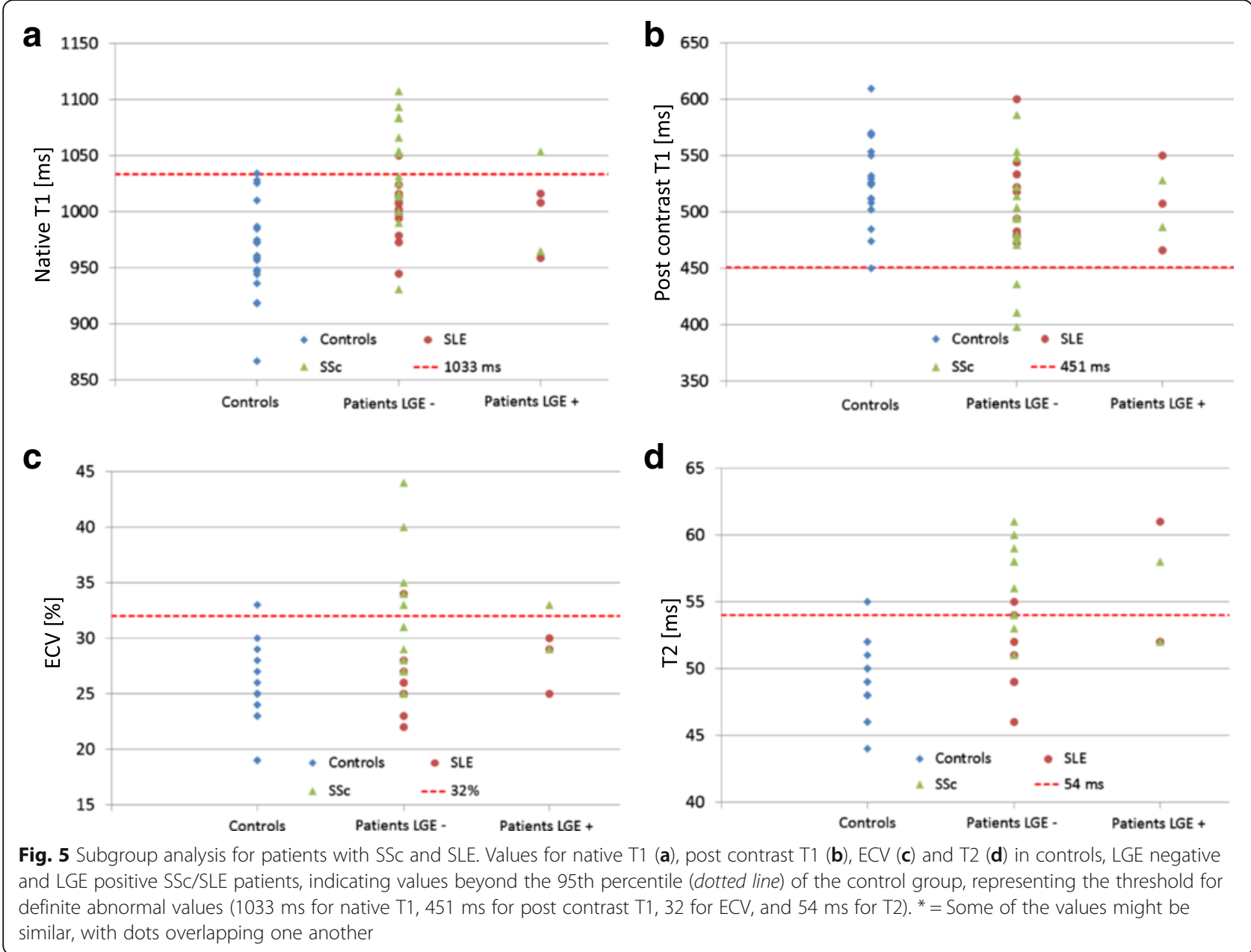

\section{Limitations}

Several potential limitations need to be addressed. Due to the single center setting, potential center-specific bias cannot be excluded. However, since most mapping sequences are vendor and center specific, there is still a lack of established normal values and thresholds, so preferably centers should establish their own normal values and thresholds upon healthy controls, as suggested by current recommendations [12].

The overall CTD group, and in particular the SSc and SLE subgroups are small, but comparable in size to most of the studies in the current literature dealing with CTD. Furthermore, despite the relatively small numbers of patients, significant differences in the mapping parameters were measured compared to controls.

Measuring global myocardial T1 or T2 values in a single mid-ventricular slice might overlook focal processes. However, this approach is common practice [33, 34], less subjective and might be even better comparable to follow-up exams. Moreover, for comparing different CMR techniques (native T1, post contrast T1, ECV, T2), it is fundamental that measurements are made in matching locations.
Endomyocardial biopsy was not routinely performed. However, it is well known that EMB has several limitations, e.g. invasiveness, sampling error, lowering its diagnostic benefit. Furthermore, in oligosymptomatic patients with preserved LV-EF, this would be a rather unethical approach, and not in line with current guidelines [35].

Comparing mapping results to cardiac biomarkers would have been of interest, however this was not intention of our study, and should be investigated by further studies.

\section{Conclusions}

We found increased values for native T1, ECV, T2, and decreased values for post contrast $\mathrm{T} 1$ in our CTD population with preserved LV-EF compared to controls, independent of the presence of LGE. Native T1, and T2 as the best discriminators to controls seem to have incremental value in the detection of myocardial involvement compared to LGE CMR alone, with the largest differences observed in patients with SSc. A potential benefit of the newer mapping techniques might be an early 
diagnosis of myocardial involvement in a still dynamic stage, yielding adequate treatment regimen, before irreversible scar (LGE) will manifest. Beside diagnosis, the new mapping techniques might be of value for monitoring of the disease.

However, further studies are mandatory, before the newer mapping sequences might be implemented in the daily clinical routine for decision-making in patients with CTD.

\section{Additional file}

Additional file 1: Details of the used T1 mapping and T2 mapping

sequences. (DOC $25 \mathrm{~kb}$ )

\section{Abbreviations}

CMR: Cardiovascular magnetic resonance; CTD: Connective tissue disorders; CVD: Cardiovascular disease; ECG: Electrocardiogram; ECV: Extracellular volume; ICD: Internal cardioverter defibrillator; IQR: Interquartile range; IVS: Interventricular septum; LA: Left atrium; LGE: Late gadolinium enhancement; LV: Left ventricle; LV-EDV: Left ventricular end-diastolic volume; LV-EF: Left ventricular ejection fraction; LV-ESV: Left ventricular endsystolic volume; MOLLI: Modified look-locker inversion recovery sequence; SCD: Sudden cardiac death; SLE: Systemic lupus erythematosus; SSc: Systemic sclerosis; SSFP: Steady-state free-precession

\section{Acknowledgements}

Not applicable.

\section{Funding}

This work was funded in part by the Robert Bosch Foundation: KKF 13-2,

KKF 15-5.

\section{Availability of supporting data}

All patients' files and results are stored in the Robert Bosch Medical Center, Stuttgart, Germany.

\section{Authors' contributions}

AM, DK, JL, SG contributed to the idea and design of the study, acquired and analyzed the data, and wrote the report. $H S, J H, F V, P K, A P, N B, D A, A G$, SG, US, HM contributed to the idea and design of the study, analysis of the data, and revision of the report. HM designed the study, contributed to the acquisition and analysis of the data, and wrote the report. All authors read and approved the final manuscript.

\section{Authors' information}

Not applicable.

\section{Competing interests}

A.G. and S.G. are employees of Siemens Healthcare GmbH. No non-financial conflicts of interest exist for any of the authors.

\section{Consent for publication}

All patients gave written informed consent for publication of their individual details and accompanying images in this manuscript. The consent form is held by the authors' institution in the patients' clinical notes, and is available for review by the Editor-in-Chief.

\section{Ethical approval and consent to participate}

The ethics committee of the University of Tuebingen approved the study and all patients gave written informed consent.

\section{Author details}

'Division of Radiology, University Hospital Innsbruck, Innsbruck, Austria. 2Division of Nephrology, Department of Internal Medicine, Robert-Bosch-Medical Center Stuttgart, Stuttgart, Germany. ${ }^{3}$ Division of Cardiology, Robert-Bosch-Medical Center Stuttgart, Auerbachstrasse 110,
70376 Stuttgart, Germany. ${ }^{4}$ Centre for Interdisciplinary Clinical Immunology, Rheumatology and Auto-inflammatory Diseases and Department of Internal Medicine II (Oncology, Haematology, Immunology, Rheumatology,

Pulmology), University Hospital Tuebingen, Tuebingen, Germany. ${ }^{5}$ Siemens Healthcare $\mathrm{GmbH}$, Erlangen, Germany.

Received: 29 June 2016 Accepted: 29 September 2016

Published online: 13 October 2016

\section{References}

1. Gaubitz M. Epidemiology of connective tissue disorders. Rheumatology. 2006;45 Suppl 3:iii3-4.

2. Puntmann VO, D'Cruz D, Smith Z, Pastor A, Choong P, Voigt T, et al. Native myocardial T1 mapping by cardiovascular magnetic resonance imaging in subclinical cardiomyopathy in patients with systemic lupus erythematosus. Circ Cardiovasc Imaging. 2013;6(2):295-301.

3. Ntusi NA, Piechnik SK, Francis JM, Ferreira VM, Rai AB, Matthews PM, et al. Subclinical myocardial inflammation and diffuse fibrosis are common in systemic sclerosis-a clinical study using myocardial T1-mapping and extracellular volume quantification. J Cardiovasc Magn Reson. 2014;16:21.

4. Follansbee WP, Curtiss El, Medsger Jr TA, Steen VD, Uretsky BF, Owens GR, et al. Physiologic abnormalities of cardiac function in progressive systemic sclerosis with diffuse scleroderma. N Engl J Med. 1984;310(3):142-8.

5. Bulkley BH, Ridolfi RL, Salyer WR, Hutchins GM. Myocardial lesions of progressive systemic sclerosis. A cause of cardiac dysfunction. Circulation. 1976;53(3):483-90.

6. Kahaleh B. The microvascular endothelium in scleroderma. Rheumatology (Oxford). 2008;47 Suppl 5:v14-5.

7. Greulich S, Ferreira VM, Dall'Armellina E, Mahrholdt H. Myocardial inflammationAre we there yet? Curr Cardiovasc Imaging Rep. 2015;8(3):6. Review.

8. Giri S, Chung YC, Merchant A, Mihai G, Rajagopalan S, Raman SV, et al. T2 quantification for improved detection of myocardial edema. J Cardiovasc Magn Reson. 2009;11:56

9. Kramer CM, Barkhausen J, Flamm SD, Kim RJ, Nagel E, Society for Cardiovascular Magnetic Resonance Board of Trustees Task Force on Standardized Protocols. Standardized cardiovascular magnetic resonance (CMR) protocols 2013 update. J Cardiovasc Magn Reson. 2013;15:91. Review.

10. Mahrholdt H, Wagner A, Holly TA, Elliott MD, Bonow RO, Kim RJ, et al. Reproducibility of chronic infarct size measurement by contrast-enhanced magnetic resonance imaging. Circulation. 2002;106(18):2322-7.

11. Mahrholdt H, Goedecke C, Wagner A, Meinhardt G, Athanasiadis A, Vogelsberg $\mathrm{H}$, et al. Cardiovascular magnetic resonance assessment of human myocarditis: a comparison to histology and molecular pathology. Circulation. 2004;109(10):1250-8.

12. Moon JC, Messroghli DR, Kellman P, Piechnik SK, Robson MD, Ugander M, Society for Cardiovascular Magnetic Resonance Imaging, Cardiovascular Magnetic Resonance Working Group of the European Society of Cardiology, et al. Myocardial T1 mapping and extracellular volume quantification: a Society for Cardiovascular Magnetic Resonance (SCMR) and CMR Working Group of the European Society of Cardiology consensus statement. J Cardiovasc Magn Reson. 2013;15:92. Review.

13. Jerosch-Herold M, Sheridan DC, Kushner JD, Nauman D, Burgess D, Dutton $D$, et al. Cardiac magnetic resonance imaging of myocardial contrast uptake and blood flow in patients affected with idiopathic or familial dilated cardiomyopathy. Am J Physiol Heart Circ Physiol. 2008;295(3):H1234-42. Epub 2008 Jul 25.

14. Mahrholdt H, Wagner A, Judd RM, Sechtem U, Kim RJ. Delayed enhancement cardiovascular magnetic resonance assessment of nonischaemic cardiomyopathies. Eur Heart J. 2005;26(15):1461-74.

15. Messroghli DR, Radjenovic A, Kozerke S, Higgins DM, Sivananthan MU, Ridgway JP. Modified Look-Locker inversion recovery (MOLLI) for highresolution T1 mapping of the heart. Magn Reson Med. 2004;52(1):141-6.

16. Arheden H, Saeed M, Higgins CB, Gao DW, Bremerich J, Wyttenbach R, et al. Measurement of the distribution volume of gadopentetate dimeglumine at echo-planar MR imaging to quantify myocardial infarction: comparison with 99mTc-DTPA autoradiography in rats. Radiology. 1999;211(3):698-708.

17. He T, Gatehouse PD, Smith GC, Mohiaddin RH, Pennell DJ, Firmin DN. Myocardial T2* measurements in iron-overloaded thalassemia: an in vivo study to investigate optimal methods of quantification. Magn Reson Med. 2008;60(5):1082-9. 
18. Valentini G, Della Rossa A, Bombardieri S, Bencivelli W, Silman AJ, D'Angelo S, et al. European multicentre study to define disease activity criteria for systemic sclerosis. II. Identification of disease activity variables and development of preliminary activity indexes. Ann Rheum Dis. 2001;60(6):592-8.

19. Gladman D, Ginzler E, Goldsmith C, Fortin P, Liang M, Urowitz M, et al. The development and initial validation of the Systemic Lupus International Collaborating Clinics/American College of Rheumatology damage index for systemic lupus erythematosus. Arthritis Rheum. 1996;39(3):363-9.

20. Pieroni M, De Santis M, Zizzo G, Bosello S, Smaldone C, Campioni M, et al. Recognizing and treating myocarditis in recent-onset systemic sclerosis heart disease: potential utility of immunosuppressive therapy in cardiac damage progression. Semin Arthritis Rheum. 2014;43(4):526-35.

21. Mavrogeni S, Douskou M, Manoussakis MN. Contrast-enhanced CMR imaging reveals myocardial involvement in idiopathic inflammatory myopathy without cardiac manifestations. JACC Cardiovasc Imaging. 2011:4(12):1324-5.

22. Steen VD, Medsger Jr TA. Severe organ involvement in systemic sclerosis with diffuse scleroderma. Arthritis Rheum. 2000;43(11):2437-44.

23. Ferreira VM, Piechnik SK, Dall'Armellina E, Karamitsos TD, Francis JM, Ntusi N, et al. T(1) mapping for the diagnosis of acute myocarditis using CMR: comparison to T2-weighted and late gadolinium enhanced imaging. JACC Cardiovasc Imaging. 2013;6(10):1048-58. Epub 2013 Sep 4.

24. Ntusi NA, Piechnik SK, Francis JM, Ferreira VM, Matthews PM, Robson MD, et al. Diffuse myocardial fibrosis and inflammation in rheumatoid arthritis: insights from CMR T1 mapping. JACC Cardiovasc Imaging. 2015;8(5):526-36. Epub 2015 Apr 15

25. Roman MJ, Salmon JE. Cardiovascular manifestations of rheumatologic diseases. Circulation. 2007;116(20):2346-55

26. Mueller KA, Mueller II, Eppler D, Zuern CS, Seizer P, Kramer U, et al. Clinical and histopathological features of patients with systemic sclerosis undergoing endomyocardial biopsy. PLoS One. 2015;10(5):e0126707.

27. Bohnen S, Radunski UK, Lund GK, Kandolf R, Stehning C, Schnackenburg B, et al. Performance of $\mathrm{t} 1$ and $\mathrm{t} 2 \mathrm{mapping}$ cardiovascular magnetic resonance to detect active myocarditis in patients with recent-onset heart failure. Circ Cardiovasc Imaging. 2015;8:6

28. Higgins CB, Herfkens R, Lipton MJ, Sievers R, Sheldon P, Kaufman L, et al. Nuclear magnetic resonance imaging of acute myocardial infarction in dogs: alterations in magnetic relaxation times. Am J Cardiol. 1983;52(1):184-8.

29. Thavendiranathan P, Walls M, Giri S, Verhaert D, Rajagopalan S, Moore S, et al. Improved detection of myocardial involvement in acute inflammatory cardiomyopathies using T2 mapping. Circ Cardiovasc Imaging. 2012;5(1): 102-10. Epub 2011 Oct 28.

30. Mavrogeni S, Sfikakis PP, Gialafos E, Bratis K, Karabela G, Stavropoulos E, et al. Cardiac tissue characterization and the diagnostic value of cardiovascular magnetic resonance in systemic connective tissue diseases. Arthritis Care Res (Hoboken). 2014;66(1):104-12.

31. Zhang Y, Corona-Villalobos CP, Kiani AN, Eng J, Kamel IR, Zimmerman SL, et al. Myocardial T2 mapping by cardiovascular magnetic resonance reveals subclinical myocardial inflammation in patients with systemic lupus erythematosus. Int J Cardiovasc Imaging. 2015:31 (2):389-97.

32. Soslow JH, Damon SM, Crum K, Lawson MA, Slaughter JC, Xu M, et al. Increased myocardial native $\mathrm{T} 1$ and extracellular volume in patients with Duchenne muscular dystrophy. J Cardiovasc Magn Reson. 2016;18(1):5.

33. Hinojar R, Varma N, Child N, Goodman B, Jabbour A, Yu CY, et al. T1 mapping in discrimination of hypertrophic phenotypes: hypertensive heart disease and hypertrophic cardiomyopathy: findings from the international T1 multicenter cardiovascular magnetic resonance study. Circ Cardiovasc Imaging. 2015;8:12.

34. Mewton N, Liu CY, Croisille P, Bluemke D, Lima JA. Assessment of myocardial fibrosis with cardiovascular magnetic resonance. J Am Coll Cardiol. 2011:57(8):891-903. Review.

35. Caforio AL, Pankuweit S, Arbustini E, Basso C, Gimeno-Blanes J, Felix SB, European Society of Cardiology Working Group on Myocardial and Pericardial Diseases, et al. Current state of knowledge on aetiology, diagnosis, management, and therapy of myocarditis: a position statement of the European Society of Cardiology Working Group on Myocardial and Pericardial Diseases. Eur Heart J. 2013;34(33):2636-48. 2648a-2648d.

\section{Submit your next manuscript to BioMed Central and we will help you at every step:}

- We accept pre-submission inquiries

- Our selector tool helps you to find the most relevant journal

- We provide round the clock customer support

- Convenient online submission

- Thorough peer review

- Inclusion in PubMed and all major indexing services

- Maximum visibility for your research

Submit your manuscript at www.biomedcentral.com/submit
Biomed Central 